\title{
An efficient model-free estimation of multiclass conditional probability
}

\author{
$\mathrm{Tu} \mathrm{Xu}$ and Junhui Wang \\ Department of Mathematics, Statistics, \\ and Computer Science \\ University of Illinois at Chicago \\ Chicago, IL 60607
}

\begin{abstract}
Conventional multiclass conditional probability estimation methods, such as Fisher's discriminate analysis and logistic regression, often require restrictive distributional model assumption. In this paper, a model-free estimation method is proposed to estimate multiclass conditional probability through a series of conditional quantile regression functions. Specifically, the conditional class probability is formulated as difference of corresponding cumulative distribution functions, where the cumulative distribution functions can be converted from the estimated conditional quantile regression functions. The proposed estimation method is also efficient as its computation cost does not increase exponentially with the number of classes. The theoretical and numerical studies demonstrate that the proposed estimation method is highly competitive against the existing competitors, especially when the number of classes is relatively large.
\end{abstract}

Keywords: interval estimate, multiclass classification, probability estimation, quantile regression, tuning

\section{Introduction}

Estimation of conditional class probability is important in statistical machine learning since the conditional class probability measures the strength and confidence of the classification outcomes. It also provides supplemental information to the classification labels, such as hazard reduction in "evidence-based" medication (Wahba, 2002) and pixel spectrum in remote sensing (Xu, 2005). In multiclass classification, a training sample $\left\{\left(\mathbf{x}_{i}, y_{i}\right) ; i=1,2, \ldots, n\right\}$ is available with covariate $\mathbf{x}_{i} \in \mathcal{R}^{p}$ and class label $y_{i} \in\{1,2, \ldots, K\}$, where $K$ is the number of classes. Due to the 
discrete feature, the conditional distribution of $Y$ given $\mathrm{X}=\mathrm{x}$ can be fully characterized by the conditional class probability $p_{k}(\mathbf{x})=P(Y=k \mid \mathbf{X}=\mathbf{x})$. Estimation of $p_{k}(\mathbf{x})$ is the primary goal of this paper, which is also known as the soft classification (Wahba, 2002; Liu, Zhang and $\mathrm{Wu}, 2011$ ), as opposed to the hard classification that mainly focuses on predicting the class labels without estimating probability.

In literature, many classical probability estimation methods have been developed based on certain distributional model assumptions. For instances, Fisher's discriminant analysis assumes that the covariates within each class follow multivariate Gaussian distributions with homogeneous or heteroscedastic covariance matrices. Relaxing the Gaussian distribution assumption, the multiple logistic regression takes one class as baseline and assumes the logarithms of all the odds ratios are linear functions of the covariates. Although these estimation methods have been widely used in practice, it is generally difficult to verify the distributional model assumptions and thus may lead to suboptimal performance when the assumptions are violated.

To circumvent the restrictive distributional assumption, various model-free probability estimation methods have been proposed and gained their popularity among the practitioners. Classification tree is a popular model-free classification method that produces probabilistic outputs, however it can be over-sensitive to the training set and thus suffers from issues of over-fitting and instability (Breiman, 1996). Wang, Shen and Liu (2008) proposes a model-free binary conditional probability estimation method by bracketing the conditional probability through a series of weighted binary large-margin classifiers with various weights $\pi \in(0,1)$. The method is based on the property that the consistent weighted binary large-margin classifiers aim at estimating $\operatorname{sign}\left(p_{1}(\mathbf{x})-\pi\right)$, and hence that the small bracket $\left(\pi, \pi^{\prime}\right)$ containing $p_{1}(\mathbf{x})$ can be obtained based on the estimated $\operatorname{sign}\left(p_{1}(\mathbf{x})-\pi\right)$ for different $\pi$ 's. To extend the binary estimation method to multiclass case, a number of attempts have been proposed. Hastie and Tibshirani (1998) and Wu, Lin and Weng (2004) develop the pairwise coupling method, which converts the multiclass probability estimation into estimating multiple one-vs-one binary conditional probabilities. Wu, Zhang and Liu (2010) 
directly extends the idea of Wang, Shen and Liu (2008) and designs an interesting way of assigning weights to the multiple classes, and then produce the estimated conditional probability by searching for the $K$-vertex polyhedron that contains $p_{k}(\mathbf{x})$. However, both methods require intensive computational cost as the number of one-vs-one binary classifications is proportional to $K^{2}$ and the number of $K$-vertex polyhedrons increases with $K$ exponentially.

In this paper, an efficient bracketing scheme is proposed for estimating the multiclass conditional probability via a series of estimated conditional quantile functions (Koenker and Bassett, 1978; Koenker, 2005). The key idea is that $p_{k}(\mathbf{x})$ can be formulated as the difference of corresponding cumulative distribution functions $P(Y \leq k \mid \mathbf{X}=\mathbf{x})$, which can be obtained through a series of estimated conditional quantiles of $Y$ given $\mathbf{X}=\mathbf{x}$. Compared with other model-free estimation methods, the proposed estimation method is computationally efficient in that its computational cost does not increase with $K$ exponentially, which is desirable especially when $K$ is large. The solution surface of the regularized quantile regression estimation (Rosset, 2009) can further alleviate the computation burden. More importantly, the asymptotic property of the proposed estimation method is established, which shows that the proposed estimation method achieves a fast convergence rate to the true $p_{k}(\mathbf{x})$. The simulation studies and real data analysis also demonstrate that the proposed method is highly competitive against the existing competitors.

The rest of the paper is organized as follows. Section 2 presents the proposed multiclass conditional probability estimation method along with its computational implementation. A tuning parameter selection criterion is also introduced. Section 3 establishes the asymptotic convergence property of the proposed method. Section 4 examines the numerical performance of the proposed estimation method in both simulated examples and real applications. Section 5 contains some discussion, and the appendix is devoted to technical proofs. 


\section{Multiclass probability estimation via quantile estimation}

This section presents the novel model-free estimation method for multiclass conditional probability and its computational implementation.

\subsection{Multiclass probability estimation via quantile regression}

In multiclass classification with $Y \in\{1, \ldots, K\}$, estimation of $p_{k}(\mathbf{x})$ is equivalent to estimation of $P(Y \leq k \mid \mathbf{X}=\mathbf{x})$ due to the following decomposition,

$$
p_{k}(\mathbf{x})=P(Y \leq k \mid \mathbf{X}=\mathbf{x})-P(Y \leq k-1 \mid \mathbf{X}=\mathbf{x}),
$$

where $P(Y \leq k \mid \mathbf{X}=\mathbf{x})=\sum_{j=1}^{k} p_{j}(\mathbf{x})$ is the conditional cumulative distribution function of $Y$ given $\mathbf{X}=\mathbf{x}$. Furthermore, the estimated $P(Y \leq k \mid \mathbf{X}=\mathbf{x})$ can be constructed through a series of estimated quantile regression functions, since

$$
P(Y \leq k \mid \mathbf{X}=\mathbf{x})=\underset{\tau}{\operatorname{argmax}}\left\{f_{\tau}^{*}(\mathbf{x}) \leq k\right\}
$$

where $f_{\tau}^{*}(\mathbf{x})$ represents the $\tau$-th conditional quantile of $Y$ given $\mathbf{X}=\mathbf{x}$, defined as

$$
f_{\tau}^{*}(\mathbf{x})=\underset{y}{\operatorname{argmin}}\{y: P(Y \leq y \mid \mathbf{X}=\mathbf{x}) \geq \tau\}
$$

Since $Y$ is discrete and only takes values in $\{1, \cdots, K\}$, estimating $f_{\tau}^{*}(\mathbf{x})$ can encounter various difficulties such as discontinuity as discussed in Machado et al. (2005) and Chen et al. (2010). A simple treatment is to jitter the discrete response by adding some continuous noises. In specific, denote the jittered response $\tilde{Y}=Y+\epsilon$, where $\epsilon$ follows a uniform distribution on $(-0.5,0.5)$ and is independent of $Y$, and denote $\tilde{f}_{\tau}^{*}(\mathbf{x})$ as the $\tau$-th quantile of $\tilde{Y}$ given $\mathbf{X}=\mathbf{x}$. With jittering, $\tilde{Y}$ becomes continuous, $P(\tilde{Y} \leq y)$ is strictly increasing in $y$, and thus $\tilde{f}_{\tau}^{*}(\mathbf{x})$ is also continuous and 
strictly increasing in $\tau$. More importantly, $P(Y \leq k)=P(\tilde{Y} \leq k+0.5)$, and

$$
f_{\tau}^{*}(\mathbf{x})=k \text { if and only if } \tilde{f}_{\tau}^{*}(\mathbf{x}) \in(k-0.5, k+0.5)
$$

Combining the results, $\tilde{f}_{\tau}^{*}(\mathbf{x})$ can be explicitly connected with $p_{k}(\mathbf{x})$ as in Lemma 1 .

Lemma 1 The $\tau$-th quantile of $\tilde{Y}=Y+\varepsilon$ given $\mathbf{X}=\mathbf{x}$ is

$$
\tilde{f}_{\tau}^{*}(\mathbf{x})=k-0.5+\frac{\tau-\sum_{j=0}^{k-1} p_{j}(\mathbf{x})}{p_{k}(\mathbf{x})}, \text { if } \sum_{j=0}^{k-1} p_{j}(\mathbf{x})<\tau \leq \sum_{j=0}^{k} p_{j}(\mathbf{x})
$$

where $p_{0}(\mathbf{x})$ is set to be 0 for simplicity.

By Lemma 1, $P(\tilde{Y} \leq k+0.5 \mid \mathbf{X}=\mathbf{x})=\underset{\tau}{\operatorname{argmax}}\left\{\tilde{f}_{\tau}^{*}(\mathbf{x}) \leq k+0.5\right\}$, and then

$$
\begin{aligned}
p_{k}(x) & =P(\tilde{Y} \leq k+0.5 \mid \mathbf{X}=\mathbf{x})-P(\tilde{Y} \leq k-0.5 \mid \mathbf{X}=\mathbf{x}) \\
& =\underset{\tau}{\operatorname{argmax}}\left\{\tilde{f}_{\tau}^{*}(\mathbf{x}) \leq k+0.5\right\}-\underset{\tau}{\operatorname{argmax}}\left\{\tilde{f}_{\tau}^{*}(\mathbf{x}) \leq k-0.5\right\} .
\end{aligned}
$$

Therefore, estimation of $p_{k}(\mathbf{x})$ boils down to estimating quantile regression function $\tilde{f}_{\tau}^{*}(\mathbf{x})$ for various $\tau$ 's. Specifically, let $0=\tau_{0}<\tau_{1}<\tau_{2}<\cdots<\tau_{m-1}<\tau_{m}=1$ be a sequence of $\tau$ 's, and $\hat{f}_{\tau_{1}}(\mathbf{x}), \hat{f}_{\tau_{2}}(\mathbf{x}), \ldots, \hat{f}_{\tau_{m-1}}(\mathbf{x})$ be the estimated $\tilde{f}_{\tau}^{*}(\mathbf{x})^{\prime}$ 's. According to $44, p_{k}(\mathbf{x})$ can be estimated as

$$
\hat{p}_{k}(\mathbf{x})=\underset{\tau_{j}}{\operatorname{argmax}}\left\{\hat{f}_{\tau_{j}}(\mathbf{x}) \leq k+0.5\right\}-\underset{\tau_{j}}{\operatorname{argmax}}\left\{\hat{f}_{\tau_{j}}(\mathbf{x}) \leq k-0.5\right\}
$$

where $\hat{f}_{\tau_{0}}(\mathbf{x})=0.5$ and $\hat{f}_{\tau_{m}}(\mathbf{x})=K+0.5$ for simplicity.

Note that $\hat{f}_{\tau}(\mathbf{x})$ can be estimated by any existing quantile regression estimation method, such as He, Ng and Portnoy (1998), Li, Liu and Zhu (2007), Wang, Zhu and Zhou (2009), Yang and He (2012), and many others. For illustration, we adopt the nonparametric method in Li, Liu and Zhu 
(2007), which is formulated as

$$
\min _{f_{\tau} \in \mathcal{H}_{K}} \sum_{i=1}^{n} \rho_{\tau}\left(y_{i}-f_{\tau}\left(\mathbf{x}_{i}\right)\right)+\frac{\lambda}{2}\left\|f_{\tau}\right\|_{\mathcal{H}_{K}}^{2},
$$

where $\mathcal{H}_{K}$ is a reproducing kernel Hilbert spaces (RKHS; Wahba 1990) induced by a pre-specified kernel function $K(\cdot, \cdot), \rho_{\tau}$ is the check loss function and $J\left(f_{\tau}\right)=\frac{1}{2}\left\|f_{\tau}\right\|_{\mathcal{H}_{K}}^{2}$ is the associated RKHS

norm. It is shown in Li, Liu, and Zhu (2007) that the estimated $\hat{f}_{\tau}(\mathbf{x})$ based on (6) converges to $\tilde{f}_{\tau}^{*}(\mathbf{x})$ in terms of $e\left(\hat{f}_{\tau}, \tilde{f}_{\tau}^{*}\right)=R\left(\hat{f}_{\tau}\right)-R\left(\tilde{f}_{\tau}^{*}\right)$ for any $\tau$, where $R\left(f_{\tau}\right)=E\left(\rho_{\tau}\left(Y-f_{\tau}(\mathbf{X})\right)\right)$.

As computational remarks, the proposed estimation method in (5) only requires fitting $m-1$ conditional quantile functions. The optimal value of $m$, as shown in Section 3, only relies on the asymptotic behavior of the quantile regression estimation. The grid points $\tau_{1}, \ldots, \tau_{m-1}$ can be simply set as equally spaced points on $(0,1)$, and more sophisticated adaptive design can be employed as well. For comparison, when the number of grid points along each direction is $m$, the computational complexity of the proposed method is $O\left(m n^{3}\right)$, whereas the complexity of the method in Wu et al. (2010) is $O\left(m^{K-1} n^{3}\right)$. It is clear that the proposed method is computationally more efficient as its complexity does not increase exponentially with $K$. Furthermore, although the true $\tilde{f}_{\tau}^{*}(\mathbf{x})$ is strictly increasing in $\tau$, the fitted quantile regression functions $\hat{f}_{\tau}(\mathbf{x})$ may cross each other and thus become inconsistent with order of $\tilde{f}_{\tau}^{*}(\mathbf{x})(\mathrm{He}, 1997)$, leading to suboptimal estimation of $\hat{p}_{k}(\mathbf{x})$ in practice. To prevent that from happening, some non-crossing constraints as in Wu and Liu (2009), Bondell, Reich and Wang (2010) and Liu and Wu (2011) can be enforced. Finally, the estimation performance of (6) largely depends on the choice of tuning parameter $\lambda$, which needs to be appropriately determined.

\subsection{Model tuning and solution surface}

In this section, a data adaptive model tuning method for multiclass conditional probability estimation is developed. To indicate the dependency on the tuning parameter $\lambda$, we denote the estimated 
conditional probability as $\hat{p}_{\lambda}(\mathbf{x})=\left(\hat{p}_{1}(\mathbf{x}), \ldots, \hat{p}_{K}(\mathbf{x})\right)^{T}$ and the quantile regression function as $\hat{f}_{\lambda, \tau}(\mathbf{x})$. The overall performance of $\hat{p}_{\lambda}(\mathbf{x})$ in estimating $p(\mathbf{x})=\left(p_{1}(\mathbf{x}), \ldots, p_{K}(\mathbf{x})\right)^{T}$ is evaluated by the generalized Kullback-Leibler (GKL) loss between $p$ and $\hat{p}_{\lambda}$,

$$
G K L\left(p, \hat{p}_{\lambda}\right)=E\left(\sum_{k=1}^{K} p_{k}(\mathbf{X}) \log \frac{p_{k}(\mathbf{X})}{\hat{p}_{k}(\mathbf{X})}\right)
$$

The corresponding comparative GKL loss, after omitting $\hat{p}_{\lambda}$-unrelated terms in (7), is

$$
G K L^{c}\left(p, \hat{p}_{\lambda}\right)=-\sum_{k=1}^{K} E\left(p_{k}(\mathbf{X}) \log \left(\hat{p}_{k}(\mathbf{X})\right)\right)
$$

It is natural to estimate $G K L^{c}\left(p, \hat{p}_{\lambda}\right)$ by its empirical version,

$$
\operatorname{EGKL}\left(\hat{p}_{\lambda}\right)=-n^{-1} \sum_{k=1}^{K} \sum_{i=1}^{n} I\left(Y_{i}=k\right) \log \hat{p}_{k}\left(\mathbf{x}_{i}\right)
$$

where $I(\cdot)$ is an indicator function. However, $E G K L\left(\hat{p}_{\lambda}\right)$ often underestimates $G K L^{c}\left(p, \hat{p}_{\lambda}\right)$ especially when the estimation model is over-complicated.

To remedy the underestimation bias, $G K L^{c}\left(p, \hat{p}_{\lambda}\right)$ can be estimated similarly as in Wang, Shen and Liu (2008) by searching for the optimal correction terms for $E G K L\left(\hat{p}_{\lambda}\right)$. Specifically, minimizing the $L_{2}$ distance between $G K L^{c}\left(p, \hat{p}_{\lambda}\right)$ and a class of candidate estimators of form $E G K L\left(\hat{p}_{\lambda}\right)+\mathbf{X}^{n}$-dependent penalty with $\mathbf{X}^{n}=\left\{\mathbf{x}_{i}\right\}_{i=1}^{n}$ yields that

$$
\widehat{G K L}^{c}\left(p, \hat{p}_{\lambda}\right)=E G K L^{c}\left(\hat{p}_{\lambda}\right)+n^{-1} \sum_{k=1}^{K} \sum_{i=1}^{n} \widehat{\operatorname{Cov}}\left(I\left(Y_{i}=k\right), \log \left(\hat{p}_{k}\left(\mathbf{x}_{i}\right)\right) \mid \mathbf{X}^{n}\right)+\widehat{D}_{n}\left(\hat{p}_{\lambda}, \mathbf{X}^{n}\right)
$$

where $D_{n}\left(\hat{p}_{\lambda}, \mathbf{X}^{n}\right)=\sum_{k=1}^{K} E\left(n^{-1} \sum_{i=1}^{n} p_{k}\left(\mathbf{x}_{i}\right) \log \left(\hat{p}_{k}\left(\mathbf{x}_{i}\right)\right)-E\left(p_{k}(\mathbf{X}) \log \left(\hat{p}_{k}(\mathbf{X})\right)\right) \mid \mathbf{X}^{n}\right)$. Here, $\operatorname{Cov}\left(I\left(Y_{i}=k\right), \log \left(\hat{p}_{k}\left(\mathbf{x}_{i}\right)\right) \mid \mathbf{X}^{n}\right)$ evaluates the accuracy of estimating $\hat{p}_{k}$ on $\mathbf{X}^{n}$, which is similar to the covariance penalty in Efron (2004) and the generalized degree of freedom in Shen and Huang (2006), and the term $D_{n}\left(\hat{p}_{\lambda}, \mathbf{X}^{n}\right)$ is a correction term adjusting the effect of random covariates $\mathbf{X}$ 
on prediction and needs to be estimated, c.f., Breiman and Spector (1992), and Breiman (1992).

To construct the estimated $\widehat{\operatorname{Cov}}\left(I\left(Y_{i}=k\right), \log \left(\hat{p}_{k}\left(x_{i}\right)\right) \mid \mathbf{X}^{n}\right)$ and $\widehat{D}_{n}\left(\hat{p}_{\lambda}, \mathbf{X}^{n}\right)$, the data perturbation technique (Wang and Shen, 2006) can be adopted. The key idea is to evaluate the generalization ability of the probability estimation method by its sensitivity to the local perturbations of $\mathbf{X}$ and $Y$. The estimation formula can be derived via derivative estimation and approximated through a Monte Carlo approximation. The exact expressions are similar to (11) and (12) in Wang, Shen and Liu (2007) and thus omitted here.

Note that the data perturbation technique requires fitting the quantile regression function multiple times for various $\tau$ 's and $\lambda$ 's, and thus can be computationally expensive. To further reduce the computation cost, the solution surface of the coefficient of $\hat{f}_{\lambda, \tau}(\mathbf{x})$ with respect to $\lambda$ and $\tau$ can be constructed following Rosset (2009). In particular, Li et al. (2007) and Takeuchi et al. (2009) show that the solution path of $\hat{f}_{\lambda, \tau}(\mathbf{x})$ is piecewise linear with respect to $\lambda$ (or $\tau$ ) when $\tau$ (or $\lambda$ ) is fixed; Rosset (2009) explores the bi-level path of regularized quantile regression and shows that the solution surface of $\hat{f}_{\lambda, \tau}(\mathbf{x})$ can be efficiently constructed with respect to both $\lambda$ and $\tau$. The solution surface is mapped as a piecewise linear function of $\tau$ or $\lambda$ and the possible locations of the bi-level optima can be found in one run of the base algorithm. That being said, the coefficient of $\hat{f}_{\lambda, \tau}(\mathbf{x})$ for various $\lambda$ 's and $\tau$ 's can be obtained at essentially the same computation cost as fitting one time of the base algorithm. Figure 1 displays $\hat{f}_{\tau, \lambda}(\mathbf{x})$ for a fixed $\mathbf{x}$ as a function of $\lambda$ and $\tau$ in a randomly selected replication of the simulated Example 1.

Figure 1 here 


\section{Statistical learning theory}

This section establishes the asymptotic convergence of the proposed multiclass conditional probability estimation method, measured by

$$
\left\|\hat{p}_{\lambda}-p\right\|_{1}=\sum_{k=1}^{K}\left\|\hat{p}_{k}-p_{k}\right\|_{1}=\sum_{k=1}^{K} E\left|\hat{p}_{k}(\mathbf{X})-p_{k}(\mathbf{X})\right|
$$

The convergence rate is quantified in terms of the tuning parameter $\lambda$, the number of brackets $m$, sample size $n$, and the cardinality of $\mathcal{F}$.

\subsection{Asymptotic theory}

The following technical assumptions are made.

Assumption 1. For any $\tau \in(0,1)$, there exists $\bar{f}_{\tau} \in \mathcal{F}$, such that $e\left(\bar{f}_{\tau}, \tilde{f}_{\tau}^{*}\right) \leq s_{n}$ for some positive sequence $s_{n} \rightarrow 0$ as $n \rightarrow \infty$.

This is analogous to Assumption 1 in Wang et al. (2008) and ensures that the true quantile regression function $\tilde{f}_{\tau}^{*}$ can be well approximated by $\mathcal{F}$.

Assumption 2. For any $\tau \in(0,1)$ and $f \in \mathcal{F}$, there exist constants $a_{1}>0$ and $0<\alpha \leq 1$ such that

$$
\left(e\left(f, \tilde{f}_{\tau}^{*}\right)\right)^{\alpha} \geq a_{1}\left\|f-\tilde{f}_{\tau}^{*}\right\|_{1}
$$

Assumption 2 describes the local smoothness of $f(\mathbf{x})$ within the neighborhood of $\tilde{f}_{\tau}^{*}(\mathbf{x})$. Note that $e\left(f, \tilde{f}_{\tau}^{*}\right)=E\left(h_{\tau}(\mathbf{X}, \tilde{Y})\right)$ with

$$
h_{\tau}(\mathbf{x}, y)=I\left(\tilde{f}_{\tau}^{*}(\mathbf{x}) \leq y \leq f(\mathbf{x})\right)(f(\mathbf{x})-y)+I\left(f(\mathbf{x}) \leq y \leq \tilde{f}_{\tau}^{*}(\mathbf{x})\right)(y-f(\mathbf{x}))
$$

by Lemma 4 in Li et al. (2007), so Assumption 2 is the same as Assumption A in Li et al. (2007).

Next we measures the cardinality of $\mathcal{F}$ by the $L_{2}$-metric entropy with bracketing. Given any 
$\epsilon>0,\left\{\left(f_{a}^{l}, f_{a}^{u}\right), a=1, \ldots, A\right\}$ is an $\epsilon$-bracketing function set of $\mathcal{F}$ if for any $f \in \mathcal{F}$ there exists an $a$ such that $f_{a}^{l} \leq f \leq f_{a}^{u}$, and $\left\|f_{a}^{l}-f_{a}^{u}\right\|_{2} \leq \epsilon$ for all $a=1, \ldots, A$. The $L_{2}$-metric entropy with bracketing $H_{B}(\epsilon, \mathcal{F})$ is then defined as the logarithm of the cardinality of the smallest $\epsilon$-bracketing function set of $\mathcal{F}$. Denote $\mathcal{F}(k)=\{f \in \mathcal{F}: J(f) \leq k\}, \mathcal{F}_{\infty}=\{f \in \mathcal{F}: J(f)<\infty\}$ and $J_{0}=\min _{\tau} \max \left\{J\left(\bar{f}_{\tau}\right), 1\right\}$.

Assumption 3. For some positive constants $a_{2}, a_{3}$ and $a_{4}$, there exists some $\epsilon_{n}>0$ such that

$$
\sup _{k \geq 1} \phi\left(\epsilon_{n}, k\right) \leq a_{2} n^{1 / 2}
$$

where $\phi\left(\epsilon_{n}, k\right)=\frac{1}{D} \int_{a_{4} D}^{a_{3}^{1 / 2} D^{\alpha / 2}} H_{B}^{1 / 2}(u, \mathcal{F}(k)) d u$ and $D=D\left(\epsilon_{n}, \lambda, k\right)=\min \left\{\epsilon_{n}^{2}+(k-1) \lambda J_{0}, 1\right\}$.

Theorem 1 Suppose Assumptions 1-3 are met, and there exists $T>0$ such that $\rho_{\tau}(y-f(\mathbf{x})) \leq T$ for any $f \in \mathcal{F}$. For $\hat{p}_{\lambda}(\mathbf{x})$ obtained as in (5),

$$
\operatorname{Pr}\left(\left\|\hat{p}_{\lambda}-p\right\|_{1} \geq \frac{4 K}{m}+2 K m^{2} a_{1}^{-1} \delta_{n}^{2 \alpha}\right) \leq 7 m K \exp \left(-a_{5} n\left(\lambda J_{0}\right)^{2-\alpha}\right),
$$

provided that $\lambda J_{0} \leq \delta_{n}^{2} / 2$, where $\delta_{n}^{2}=\min \left\{\max \left(\epsilon_{n}^{2}, s_{n}\right), 1\right\}$.

Corollary 1 Under the assumptions in Theorem 1,

$$
\left\|\hat{p}_{\lambda}-p\right\|_{1}=O_{p}\left(\frac{2}{m}+m^{2} a_{1}^{-1} \delta_{n}^{2 \alpha}\right), \quad E\left\|\hat{p}_{\lambda}-p\right\|_{1}=O\left(\frac{2}{m}+m^{2} a_{1}^{-1} \delta_{n}^{2 \alpha}\right)
$$

provided that $n\left(\lambda J_{0}\right)^{2-\alpha}-\log (m)$ diverges as $n \rightarrow \infty$.

Theorem 1 and Corollary 1 provide probability and risk bounds for $\left\|\hat{p}_{\lambda}-p\right\|_{1}$. They also suggest the ideal $m$ to be of order $O\left(\delta_{n}^{-2 \alpha / 3}\right)$, yielding the fast rate of $O_{p}\left(\delta_{n}^{2 \alpha / 3}\right)$ for $\left\|\hat{p}_{\lambda}-p\right\|_{1}$. 


\subsection{A theoretic example}

To illustrate the asymptotic theory, a simple theoretic example is considered. Let $\mathbf{X}$ be sampled from a uniform distribution on $(0,3)$ and $Y \in\{1,2,3\}$ be sampled according to $p_{k}(x)=0.8$ if $k-1 \leq x<k$ and 0.1 otherwise. Let $\mathcal{F}_{1}=\left\{f: f \in \mathcal{H}_{K}, f(x) \in(0.5,3.5)\right\}$, where $K$ is the Gaussian kernel.

To verify Assumption 1 , note that for any $\tau \in(0,1), \tilde{f}_{\tau}^{*}(x)$ is continuous in $x$ except at $x=1$ and $x=2$. For given $s_{n}$, define

$g_{\tau}(x)= \begin{cases}\tilde{f}_{\tau}^{*}\left(k-\frac{s_{n}}{8}\right)+\frac{x-\left(k-\frac{s_{n}}{8}\right)}{\frac{s_{n}}{4}}\left(\tilde{f}_{\tau}^{*}\left(k+\frac{s_{n}}{8}\right)-\tilde{f}_{\tau}^{*}\left(k-\frac{s_{n}}{8}\right)\right), & \text { if } x \in\left(k-\frac{s_{n}}{8}, k+\frac{s_{n}}{8}\right), k=1,2 ; \\ \tilde{f}_{\tau}^{*}(x), & \text { otherwise, }\end{cases}$ then $g_{\tau}(x)$ is a continuous function of $x$, and $\left\|g_{\tau}-\tilde{f}_{\tau}^{*}\right\|_{1} \leq s_{n} / 2$. Furthermore, as $g_{\tau}(x)$ is continuous, Steinwart (2001) shows that there exists a $\bar{f}_{\tau} \in \mathcal{F}_{1}$ such that $\left\|g_{\tau}-\bar{f}_{\tau}\right\|_{1} \leq \| g_{\tau}-$ $\bar{f}_{\tau} \|_{\infty} \leq s_{n} / 2$. Therefore, $\left\|\bar{f}_{\tau}-\tilde{f}_{\tau}^{*}\right\|_{1} \leq\left\|g_{\tau}-\tilde{f}_{\tau}^{*}\right\|_{1}+\left\|g_{\tau}-\bar{f}_{\tau}\right\|_{1} \leq s_{n}$. Since $\mid \rho_{\tau}\left(y-\bar{f}_{\tau}(x)\right)-$ $\rho_{\tau}\left(y-\tilde{f}_{\tau}^{*}(x)\right)|\leq|\left(y-\bar{f}_{\tau}(x)\right)-\left(y-\tilde{f}_{\tau}^{*}(x)\right)|=| \bar{f}_{\tau}(x)-\tilde{f}_{\tau}^{*}(x) \mid$, then

$$
e\left(\bar{f}_{\tau}, \tilde{f}_{\tau}^{*}\right)=E\left(\rho_{\tau}\left(\tilde{Y}-\bar{f}_{\tau}(X)\right)-\rho_{\tau}\left(\tilde{Y}-\tilde{f}_{\tau}^{*}(X)\right)\right) \leq E\left|\bar{f}_{\tau}(X)-\tilde{f}_{\tau}^{*}(X)\right|=\left\|\bar{f}_{\tau}-\tilde{f}_{\tau}^{*}\right\|_{1} \leq s_{n} .
$$

To verify Assumption 2, note that $e\left(f, \tilde{f}_{\tau}^{*}\right)=E\left(h_{\tau}(X, \tilde{Y})\right)=E\left(E\left(h_{\tau}(X, \tilde{Y}) \mid X\right)\right)$, and

$$
\begin{aligned}
& E\left(h_{\tau}(X, \tilde{Y}) \mid X\right) \\
= & E\left(I\left(\tilde{f}_{\tau}^{*}(X) \leq y \leq f(X)\right)(f(X)-\tilde{Y})+I\left(f(X) \leq \tilde{Y} \leq \tilde{f}_{\tau}^{*}(X)\right)(\tilde{Y}-f(X)) \mid X\right) \\
= & \left|\int_{\tilde{f}_{\tau}^{*}(X)}^{f(X)} P_{X}(u)(f(X)-u) d u\right| \geq 0.1\left|\int_{\tilde{f}_{\tau}^{*}(X)}^{f(X)}(f(X)-u) d u\right|=0.05\left|\tilde{f}_{\tau}^{*}(X)-f(X)\right|^{2},
\end{aligned}
$$

where $P_{X}(u)=p_{k}(X)$ if $k-0.5 \leq u<k+0.5$. Therefore, Assumption 2 is satisfied with $\alpha=0.5$ and $a_{1}=\sqrt{0.05}$. 
To verify Assumption 3, since $\mathcal{H}_{B}(u, \mathcal{F}(k))=O\left(\log ^{2}(k / u)\right)$ (Zhou, 2002) for any given $k$ and $\phi\left(\epsilon_{n}, k\right)$ is nonincreasing in $D$, there exist positive constants $c_{1}, c_{2}$, such that

$$
\sup _{k \geq 1} \phi\left(\epsilon_{n}, k\right) \leq \phi\left(\epsilon_{n}, 1\right)=\frac{1}{D} \int_{a_{4} D}^{a_{3}^{1 / 2} D^{\alpha / 2}} c_{1} \log (1 / u) d u \leq c_{2} \log \left(1 / \epsilon_{n}\right) / \epsilon_{n}^{2-\alpha}
$$

Without loss of generality, assume $s_{n} \leq \epsilon_{n}^{2} \leq 1$, and then $\delta_{n}^{2}=\epsilon_{n}^{2}$. Solving (9), yields that $\delta_{n}^{2}=O\left(\left(\frac{\log ^{2} n}{n}\right)^{1 /(2-\alpha)}\right)$, when $\lambda J_{0} \sim \delta_{n}^{2}$.

Finally, by Corollary $1, E\left\|\hat{p}_{\lambda}-p\right\|_{1}=O\left(\frac{2}{m}+m^{2} a_{1}^{-1} n^{-1 / 3}(\log n)^{2 / 3}\right)$. This implies that $E\left\|\hat{p}_{\lambda}-p\right\|_{1}=O\left(n^{-1 / 9}(\log n)^{2 / 9}\right)$ when $m$ is set as $O\left(n^{1 / 9}(\log n)^{-2 / 9}\right)$.

\section{Numerical experiments}

This section examines the effectiveness of the proposed multiclass probability estimation method in simulated and real examples. The numerical performance of the proposed method (OUR) is compared against three popular competitors: baseline logistic model (BLM), classification tree (TREE) and weighted multiclass classification (WMC; Wu et al., 2010). For illustration, the number of quantiles $m$ in our method is set as $m=100$. The kernel function used in each method is set as the Gaussian kernel $K\left(\mathbf{z}_{1}, \mathbf{z}_{2}\right)=e^{-\left\|\mathbf{z}_{1}-\mathbf{z}_{2}\right\|^{2} / 2 \sigma^{2}}$, where the scale parameter $\sigma^{2}$ is set as the median of pairwise Euclidean distances within the training set. To optimize the performance of each estimation method, a grid search is employed to select the tuning parameter as in Section 2.2. The grid used in all examples is set as $\left\{10^{(s-31) / 10} ; s=1, \ldots, 61\right\}$. A more refined grid search can be employed to further improve the numerical performance at the cost of increased computation burden.

In simulated examples where the true conditional probability $p_{k}(\mathbf{x})$ is known, the performance of each estimation method is measured by its distance to $p_{k}(\mathbf{x})$. Various distance measures between 
$\hat{p}_{k}(\mathbf{x})$ and $p_{k}(\mathbf{x})$ are computed based on the testing set,

$$
\begin{aligned}
& \text { 1-Norm error: } \operatorname{err}_{1}\left(\hat{p}_{\lambda}, p\right)=\frac{1}{|T|} \sum_{t \in T} \sum_{k=1}^{K}\left|\hat{p}_{k}\left(\mathbf{x}_{t}\right)-p_{k}\left(\mathbf{x}_{t}\right)\right| ; \\
& \text { 2-Norm error: } \operatorname{err}_{2}\left(\hat{p}_{\lambda}, p\right)=\frac{1}{|T|} \sum_{t \in T} \sum_{k=1}^{K}\left(\hat{p}_{k}\left(\mathbf{x}_{t}\right)-p_{k}\left(\mathbf{x}_{t}\right)\right)^{2} ; \\
& \text { GKL loss: } \quad \operatorname{err}_{K L}\left(\hat{p}_{\lambda}, p\right)=\frac{1}{|T|} \sum_{t \in T} \sum_{k=1}^{K} p_{k}\left(\mathbf{x}_{t}\right) \log \frac{p_{k}\left(\mathbf{x}_{t}\right)}{\hat{p}_{k}\left(\mathbf{x}_{t}\right)} ; \\
& \text { Cross entropy error (CEE): } \quad \operatorname{err}_{C E}\left(\hat{p}_{\lambda}\right)=\frac{1}{|T|} \sum_{t \in T}-\log \left(\hat{p}_{y_{t}}\left(\mathbf{x}_{t}\right)\right)
\end{aligned}
$$

where $T$ denotes the testing set, and $|T|$ is the cardinality of $T$. To avoid degeneration in computing GKL loss and CEE, a small correction constant 0.01 is added to $\hat{p}_{k}(\mathbf{x})$ when necessary.

\subsection{Simulated examples}

Five simulated examples are generated for comparison.

Example 1. First, $Y$ is generated uniformly over $\{1,2,3,4,5\}$. Next, given $Y=y$, the covariates $\mathbf{X}$ are generated from $\mathrm{T}(\mu(y), \Sigma, d f=2)$, a multivariate $t$ distribution with $\mu(y)=$ $(\cos (2 y \pi / 5), \sin (2 y \pi / 5))^{T}, \Sigma=\operatorname{diag}(1,2)$ and degree of freedom 2. The training size is 400, and the testing size is 2600 .

Example 2. First, $Y$ is generated uniformly over $\{1,2, \ldots, 10\}$. Next, given $Y=y$, the covariates $\mathbf{X}$ are generated from $\mathrm{T}(\mu(y), \Sigma, d f=2)$, where $\mu(y)=(\cos (y \pi / 5), \sin (y \pi / 5))^{T}$ and $\Sigma=\operatorname{diag}(1,2)$. The training size is 400 , and the testing size is 2600 .

Example 3. First, $Y$ is generated uniformly over $\{1,2, \ldots, 20\}$. Next, given $Y=y$, the covariates $\mathbf{X}$ are generated from $\mathrm{T}(\mu(y), \Sigma, d f=2)$, where $\mu(y)=(\cos (y \pi / 10), \sin (y \pi / 10))^{T}$ and $\Sigma=\operatorname{diag}(1,2)$. The training size is 400 , and the testing size is 2600 .

Example 4. First, $Y$ is generated uniformly over $\{1,2,3,4,5\}$. Next, given $Y=y$, the covariates $\mathbf{X}$ are generated from $\mathrm{T}(\mu(y), \Sigma, d f=2)$, where $\mu(y)=(\cos (2 y \pi / 5), \sin (2 y \pi / 5), 0, \ldots, 0)^{T}$, 
and $\Sigma=\operatorname{diag}(1,2,1, \ldots, 1)$ if $y$ is odd and $\Sigma=\operatorname{diag}(2,1,1, \ldots, 1)$ if $y$ is even. The training size is 400 , and the testing size is 2600 .

Example 5. First, $Y$ is generated uniformly over $\{1,2, \ldots, 10\}$. Next, given $Y=y$, the covariates $\mathbf{X}$ are generated from $\mathrm{T}(\mu(y), \Sigma, d f=2)$, where $\mu(y)=(\cos (y \pi / 5), \sin (y \pi / 5), 0, \ldots, 0)^{T}$, and $\Sigma=\operatorname{diag}(1,2,1, \ldots, 1)$ if $y$ is odd and $\Sigma=\operatorname{diag}(2,1,1, \ldots, 1)$ if $y$ is even. The training size is 400 , and the testing size is 2600 .

Examples 1-3 are generated similarly, but with different number of classes $K$ and different mean vectors $\mu(y)$. When $K$ gets larger, the generated data from different classes become more overlapped and thus the resultant classification becomes more difficult. Examples 4 and 5 include additional noise variables and heteroscedastic covariance matrices. Each simulated example is repeated 50 times, and the averaged test errors and the corresponding standard deviations are reported in Table 1.

Table 1 here

Evidently, the proposed estimation method delivers superior numerical performance, and outperforms BLM, TREE and WMC in all the examples. As a model-free method, WMC yields competitive performance in Example 1 and Example 4 with $K=5$ where the data from different classes are relatively far apart leading to clear-cut classification boundary. However, when $K$ gets larger, WMC requires much more intensive computing power, and its numerical performance appears to be less satisfactory in Examples 2 and 5 with $K=10$. Furthermore, the performance of WMC in Example 3 with $K=20$ is not reported in Table 1, since it is computationally expensive to achieve reasonably good estimation accuracy.

\subsection{Real applications}

In this section, the proposed multiclass probability estimation method is applied to the iris data, the white wine quality data and the abalone data. All datasets are publicly available at the University 
of California Irvine Machine Learning Repository (http://archive.ics.uci.edu/ml/).

The iris data has 4 continuous attributes: sepal length, sepal width, petal length, and petal width, and three classes: Setosa, Versicolour, and Virginica. The size of the iris dataset is 150, and each class has 50 observations. We randomly select 30 observations from each class and set as the training set, and the remaining 60 observations are used for testing. The white wine quality data has 11 attributes, which characterize various aspects of the white wines, and the response ranges from 0 to 10 representing quality scores made by wine experts. For illustration, we focus only on three classes with quality scores 5, 6 and 7, and a total of 4535 white wines are selected, where 1457, 2198 and 880 white wines score 5, 6 and 7, respectively. We randomly select 100 white wines from each class as the training set, and the remaining 4235 white wines are used for testing. The abalone data has 8 attributes on various physical measurements of an abalone, and 29 classes representing different ages of an abalone. Since some extreme classes have very few abalones, we only focus on the $K$ largest classes with $K=5,8,10$. In specific, for $K=5$, classes $7-11$ are selected with a total of 2768 abalones; for $K=8$, classes $6-13$ are selected with a total of 3498 abalones; for $K=10$, classes $5-14$ are selected with a total of 3739 abalones. In all scenarios, we randomly select 50 abalones from each class as the training set, and keep the remaining abalones for testing.

Note that the true conditional probability $p_{k}(\mathbf{x})$ is not available in the real applications, so only CEE is computed and used for comparison. In addition, we also compare the averaged misclassification error (MCE) of each probability estimation method on the testing set, where the classification label is predicted as $\hat{y}_{t}=\operatorname{argmax}_{k} \hat{p}_{k}\left(\mathbf{x}_{t}\right)$, and MCE is defined as

$$
\operatorname{MCE}\left(\hat{p}_{\lambda}, p\right)=\frac{1}{|T|} \sum_{t \in T} I\left(\hat{y}_{t} \neq y_{t}\right) .
$$

The averaged CEE and MCE over 50 replications are reported in Table 2.

Table 2 here 
It is evident that the proposed probability estimation method delivers competitive results against other competitors. It yields the smallest CEE and MCE in all real examples, except that WMC produces slightly smaller CEE in the iris example. The performance of WMC is not reported for the abalone example with $K=8$ and 10 due to the computational burden.

\section{Summary}

This paper proposes an efficient model-free multiclass conditional probability estimation method, where the estimated probabilities are constructed via a series of estimated conditional quantile regression functions. The proposed method does not require any distributional model assumption, and it is computationally efficient as its computation cost does not need to increase exponentially with $K$. The asymptotic convergence rate of the proposed method is established, and the numerical experiments with both simulated examples and real applications demonstrate the advantage of the proposed method, especially when $K$ is large. In addition, $p_{k}(\mathbf{x})=P(Y=k \mid \mathbf{x})$ can be regarded as the conditional density of discrete $Y$, and thus the proposed method can be naturally extended to a general framework of conditional density estimation (Hansen, 2004).

\section{Appendix: technical proofs}

Proof of Lemma 1. When $\sum_{j=1}^{k-1} p_{j}(\mathbf{x})<\tau \leq \sum_{j=1}^{k} p_{j}(\mathbf{x})$,

$$
\begin{aligned}
P\left(\tilde{Y} \leq \tilde{f}_{\tau}^{*}(\mathbf{x})\right) & =P(Y \leq k-1)+\operatorname{Pr}\left(Y=k,-0.5 \leq \varepsilon \leq \frac{\tau-\sum_{j=1}^{k-1} p_{j}(\mathbf{x})}{p_{k}(\mathbf{x})}-0.5\right) \\
& =\sum_{j=1}^{k-1} p_{j}(\mathbf{x})+p_{k}(x) \times \frac{\tau-\sum_{j=1}^{k-1} p_{j}(\mathbf{x})}{p_{k}(\mathbf{x})}=\tau .
\end{aligned}
$$


The desired result follows immediately.

Proof of Theorem 1. First, note that $p_{k}(\mathbf{x})=\sum_{s=0}^{k} p_{s}(\mathbf{x})-\sum_{s=0}^{k-1} p_{s}(\mathbf{x})$ with $p_{0}(\mathbf{x})=0$, and then

$$
\begin{aligned}
\left\|\hat{p}_{k}-p_{k}\right\|_{1} & =\left\|\sum_{s=0}^{k} \hat{p}_{s}-\sum_{s=0}^{k-1} \hat{p}_{s}-\sum_{s=0}^{k} p_{s}+\sum_{s=0}^{k-1} p_{s}\right\|_{1} \\
& \leq\left\|\sum_{s=0}^{k} \hat{p}_{s}-\sum_{s=0}^{k} p_{s}\right\|_{1}+\left\|\sum_{s=0}^{k-1} \hat{p}_{s}-\sum_{s=0}^{k-1} p_{s}\right\|_{1} .
\end{aligned}
$$

Therefore, it suffices to bound $\left\|\sum_{s=0}^{k} \hat{p}_{s}-\sum_{s=0}^{k} p_{s}\right\|_{1}$ for any $k$.

Next, for simplicity, denote $\hat{P}_{k}(\mathbf{x})=\sum_{s=0}^{k} \hat{p}_{s}(\mathbf{x}), P_{k}(\mathbf{x})=\sum_{s=0}^{k} p_{s}(\mathbf{x})$, and $B_{k}=\left\{\mathbf{x}: \mid \hat{P}_{k}(\mathbf{x})-\right.$ $\left.P_{k}(\mathbf{x}) \mid \geq \frac{2}{m}\right\}$. Simple calculation yields that

$$
\begin{aligned}
\left\|\hat{P}_{k}-P_{k}\right\|_{1} & =E\left|\hat{P}_{k}(\mathbf{X})-P_{k}(\mathbf{X})\right| \\
& =E\left(\left|\hat{P}_{k}(\mathbf{X})-P_{k}(\mathbf{X})\right| \cdot I\left(B_{k}\right)\right)+E\left(\left|\hat{P}_{k}(\mathbf{X})-P_{k}(\mathbf{X})\right| \cdot I\left(B_{k}^{c}\right)\right) \\
& \leq P\left(B_{k}\right)+\frac{2}{m} P\left(B_{k}^{c}\right) \leq P\left(B_{k}\right)+\frac{2}{m}
\end{aligned}
$$

where the first inequality follows from the fact that $\left|\hat{P}_{k}(\mathbf{X})-P_{k}(\mathbf{X})\right|$ is bounded by 1 . Therefore, bounding $\left\|\hat{P}_{k}-P_{k}\right\|_{1}$ boils down to bounding $P\left(B_{k}\right)$.

Based on the estimation method in 5 , there exists $j_{1} \in\{1, \ldots, m-1\}$, such that $\tau_{j_{1}}=\hat{P}_{k}(\mathbf{x})$, and then $\hat{f}_{\tau_{j_{1}}}(\mathbf{x}) \leq k+0.5$ and $\hat{f}_{\tau_{j_{1}+1}}(\mathbf{x})>k+0.5$. Let $\triangle_{j}=\left\{\mathbf{x}:\left|\hat{f}_{\tau_{j}}(\mathbf{x})-\tilde{f}_{\tau_{j}}^{*}(\mathbf{x})\right| \geq \frac{1}{m}\right\}$, and we will show the relationship $B_{k}=\left\{\mathbf{x}:\left|\hat{P}_{k}(\mathbf{x})-P_{k}(\mathbf{x})\right| \geq \frac{2}{m}\right\} \subset \bigcup_{j=1}^{m-1} \triangle_{j}$ in the following four cases.

Case 1. If $\hat{P}_{k}(\mathbf{x})-P_{k}(\mathbf{x}) \geq \frac{2}{m}$ and $\hat{P}_{k}(\mathbf{x}) \leq P_{k+1}(\mathbf{x})$, then $P_{k}(\mathbf{x})+\frac{2}{m} \leq \tau_{j_{1}}=\hat{P}_{k}(\mathbf{x}) \leq$ $P_{k+1}(\mathbf{x})$. Based on Lemma 1,

$$
\tilde{f}_{\tau_{j_{1}}}^{*}(\mathbf{x})=k+0.5+\frac{\hat{P}_{k}(\mathbf{x})-P_{k}(\mathbf{x})}{p_{k+1}(\mathbf{x})} \geq k+0.5+\hat{P}_{k}(\mathbf{x})-P_{k}(\mathbf{x}) \geq k+0.5+\frac{2}{m},
$$


which implies that $\tilde{f}_{\tau_{j_{1}}}^{*}(\mathbf{x})-\hat{f}_{\tau_{j_{1}}}(\mathbf{x}) \geq \frac{2}{m}>\frac{1}{m}$.

Case 2. If $\hat{P}_{k}(\mathbf{x})-P_{k}(\mathbf{x}) \geq \frac{2}{m}$ and $\hat{P}_{k}(\mathbf{x})>P_{k+1}(\mathbf{x})$, then by Lemma $1, \tilde{f}_{\tau_{j_{1}}}^{*}(\mathbf{x})>k+1.5$ and $\tilde{f}_{\tau_{j_{1}}}^{*}(\mathbf{x})-\hat{f}_{\tau_{j_{1}}}(\mathbf{x})>1>\frac{1}{m}$.

Case 3. If $P_{k}(\mathbf{x})-\hat{P}_{k}(\mathbf{x}) \geq \frac{2}{m}$ and $\hat{P}_{k}(\mathbf{x})>P_{k-1}(\mathbf{x})-\frac{1}{m}$, then $P_{k-1}(\mathbf{x})-\frac{1}{m}<\tau_{j_{1}} \leq P_{k}(\mathbf{x})-\frac{2}{m}$ and $P_{k-1}(\mathbf{x})<\tau_{j_{1}+1}=\tau_{j_{1}}+\frac{1}{m} \leq P_{k}(\mathbf{x})-\frac{1}{m}$. Based on Lemma 1,

$$
\tilde{f}_{\tau_{j_{1}+1}}^{*}(\mathbf{x})=k-0.5+\frac{\tau_{j_{1}+1}-P_{k-1}(\mathbf{x})}{p_{k}(\mathbf{x})} \leq k-0.5+\frac{p_{k}(\mathbf{x})-\frac{1}{m}}{p_{k}(\mathbf{x})} \leq k+0.5-\frac{1}{m}
$$

which implies that $\hat{f}_{\tau_{j_{1}+1}}-\tilde{f}_{\tau_{j_{1}+1}}^{*}>\frac{1}{m}$.

Case 4. If $P_{k}(\mathbf{x})-\hat{P}_{k}(\mathbf{x}) \geq \frac{2}{m}$ and $\hat{P}_{k}(\mathbf{x}) \leq P_{k-1}(\mathbf{x})-\frac{1}{m}$, then $\tau_{j_{1}+1} \leq P_{k-1}(\mathbf{x})$ and by Lemma $1, \tilde{f}_{\tau_{j_{1}+1}}^{*}(\mathbf{x}) \leq k-0.5$ and $\hat{f}_{\tau_{j_{1}+1}}(\mathbf{x})-\tilde{f}_{\tau_{j_{1}+1}}^{*}(\mathbf{x})>1>\frac{1}{m}$.

Combining the above four cases, $B_{k} \subset \bigcup_{j=1}^{m-1} \triangle_{j}=\left\{\mathbf{x}:\left|\hat{f}_{\tau_{j}}(\mathbf{x})-\tilde{f}_{\tau_{j}}^{*}(\mathbf{x})\right| \geq \frac{1}{m}\right.$ for some $\left.j\right\}$. It leads to a connection between $\left\|\hat{P}_{k}-P_{k}\right\|_{1}$ and $e\left(\hat{f}_{\tau}, \tilde{f}_{\tau}^{*}\right)$ is established in the following.

$$
\begin{aligned}
& \left\{\left\|\hat{P}_{k}-P_{k}\right\|_{1} \geq \frac{2}{m}+m^{2} a_{1}^{-1} \delta_{n}^{2 \alpha}\right\} \subset\left\{P\left(B_{k}\right) \geq m^{2} a_{1}^{-1} \delta_{n}^{2 \alpha}\right\} \\
\subset & \left\{\operatorname{Pr}\left(\bigcup_{j=1}^{m-1} \triangle_{j}\right) \geq m^{2} a_{1}^{-1} \delta_{n}^{2 \alpha}\right\} \subset\left\{P\left(\triangle_{j}\right) \geq m a_{1}^{-1} \delta_{n}^{2 \alpha}, \text { for some } \mathrm{j}\right\} .
\end{aligned}
$$

Therefore, $\operatorname{Pr}\left(\left\|\hat{P}_{k}-P_{k}\right\|_{1} \geq \frac{2}{m}+m^{2} a_{1}^{-1} \delta_{n}^{2 \alpha}\right) \leq \sum_{j=1}^{m-1} \operatorname{Pr}\left(P\left(\triangle_{j}\right) \geq m a_{1}^{-1} \delta_{n}^{2 \alpha}\right)$. In addition, $P\left(\triangle_{j}\right) \geq m a_{1}^{-1} \delta_{n}^{2 \alpha}$ implies that $\left\|\hat{f}_{\tau_{j}}-\tilde{f}_{\tau_{j}}^{*}\right\|_{1} \geq \frac{1}{m} P\left(\triangle_{j}\right)=a_{1}^{-1} \delta_{n}^{2 \alpha}$. This, together with Assumption 2 , yields that $e\left(\hat{f}_{\tau_{j}}, \tilde{f}_{\tau_{j}}^{*}\right) \geq \delta_{n}^{2}$. Therefore,

$$
\begin{aligned}
& \operatorname{Pr}\left(\left\|\hat{P}_{k}-P_{k}\right\|_{1} \geq \frac{2}{m}+m^{2} a_{1}^{-1} \delta_{n}^{2 \alpha}\right) \leq \sum_{j=1}^{m-1} \operatorname{Pr}\left(P\left(\triangle_{j}\right) \geq m a_{1}^{-1} \delta_{n}^{2 \alpha}\right) \\
\leq & \sum_{j=1}^{m-1} \operatorname{Pr}\left(e\left(\hat{f}_{\tau_{j}}, \tilde{f}_{\tau_{j}}^{*}\right) \geq \delta_{n}^{2}\right) \leq m \cdot \max _{j}\left\{3.5 \exp \left(-a_{5} n\left(\lambda J_{\tau_{j}}\right)^{2-\alpha}\right)\right\} \\
\leq & 3.5 m \exp \left(-a_{5} n\left(\lambda J_{0}\right)^{2-\alpha}\right),
\end{aligned}
$$


where the second to the last inequality follow from a slightly modified version of Theorem 2 in $\mathrm{Li}$ et al. (2007) incorporating the approximation error in Assumption 1.

Based on 11, $\left\|\hat{p}_{k}-p_{k}\right\|_{1} \geq \frac{4}{m}+2 m^{2} a_{1}^{-1} \delta_{n}^{2 \alpha}$ implies that at least one of $\left\|\hat{P}_{k}-P_{k}\right\|_{1}$ and $\left\|\hat{P}_{k-1}-P_{k-1}\right\|_{1}$ is larger than $\frac{2}{m}+m^{2} a_{1}^{-1} \delta_{n}^{2 \alpha}$. Therefore,

$$
\begin{aligned}
& \operatorname{Pr}\left(\left\|\hat{p}_{\lambda}-p\right\|_{1} \geq \frac{4 K}{m}+2 K m^{2} a_{1}^{-1} \delta_{n}^{2 \alpha}\right) \\
\leq & \operatorname{Pr}\left(\bigcup_{k=1}^{K}\left\{\left\|\hat{p}_{k}-p_{k}\right\|_{1} \geq \frac{4}{m}+2 m^{2} a_{1}^{-1} \delta_{n}^{2 \alpha}\right\}\right) \leq \sum_{k=1}^{K} \operatorname{Pr}\left(\left\|\hat{p}_{k}-p_{k}\right\|_{1} \geq \frac{4}{m}+2 m^{2} a_{1}^{-1} \delta_{n}^{2 \alpha}\right) \\
\leq & \sum_{k=1}^{K} \operatorname{Pr}\left(\left\|\hat{P}_{k}-P_{k}\right\|_{1} \geq \frac{2}{m}+m^{2} a_{1}^{-1} \delta_{n}^{2 \alpha}\right)+\sum_{k=1}^{K} \operatorname{Pr}\left(\left\|\hat{P}_{k-1}-P_{k-1}\right\|_{1} \geq \frac{2}{m}+m^{2} a_{1}^{-1} \delta_{n}^{2 \alpha}\right) \\
\leq & 7 m K \exp \left(-a_{5} n\left(\lambda J_{0}\right)^{2-\alpha}\right) .
\end{aligned}
$$

\section{References}

[1] Bondell, H., Reich, B. And WANG, H. (2010). Non-crossing quantile regression curve estimation, Biometrika, 97, 825-838.

[2] BREIMAN, L. (1992). The little bootstrap and other methods for dimensionality selection in regression: X-fixed Prediction Error, Journal of the American Statistical Association, 87, 738-754.

[3] Breiman, L. ANd Spector, P. (1992). Submodel selection and evaluation in regression the X- random case, International Statistical Review, 3, 291-319.

[4] Breiman, L. (1996). Heuristics of instability and stabilization in model selection, Annals of Statistics, 26, 801-849.

[5] Chen, J. And LAZAR, N. (2010). Quantile estimation for discrete data via empirical likelihood, Journal of Nonparametric Statistics, 22, 237-255. 
[6] EFRON, B. (2004). The estimation of prediction error: covariance penalties and crossvalidation, Journal of the American Statistical Association, 99, 619-632.

[7] Gu, C. (2002). Smoothing spline ANOVA models, New York: Springer-Verlag.

[8] Hansen, B. (2004). Nonparametric conditional density estimation, Unpublished manuscript.

[9] Hastie, T. And Tibshirani, R. (1998). Classification by pairwise coupling, The Annals of Statistics, 26, 451-471.

[10] HE, X. (1997). Quantile curves without crossing, The American Statistician, 51, 186-192.

[11] He, X., NG, P. And Portnoy S. (1998). Bivariate quantile smoothing splines, Journal of the Royal Statistical Society, Series B, 60, 537-550.

[12] Kimeldorf, G. And Wahba, G. (1971). Some results on Tchebycheffian spline functions, Journal of Mathematical Analysis and Applications, 33, 82-95.

[13] Koenker, R. (2005). Quantile regression, New York: Cambridge University Press.

[14] Koenker, R. And Bassett, G. (1978). Regression quantiles, Econometrica, 46, 33-50.

[15] LEE, Y., LiN, Y. AND WAHBA, G. (2004). Multicategory support vector machines, theory, and application to the classification of micoarray data and satellite radiance data, Journal of the American Statistical Association, 99, 67-81.

[16] Li, Y., LiU, Y. AND ZHU, J. (2007). Quantile regression in reproducing kernel Hilbert spaces, Journal of the American Statistical Association, 102, 255-268.

[17] LIU, Y. AND WU, Y. (2011). Simultaneous multiple non-crossing quantile regression estimation using kernel constraints, Journal of Nonparametric Statistics, 23, 415-437. 
[18] LiU, Y., Zhang, H. H. AND Wu, Y. (2011). Soft and hard classification? Large-margin unified machines, Journal of the American Statistical Association, 106, 166-177.

[19] Machado, J. And Santos Silva, J. (2005). Quantiles for counts, Journal of American Statistical Association, 100, 1226-1237.

[20] Rosset, S. (2009). Bi-level path following for cross validated solution of kernel quantile regression, Journal of Machine Learning Research, 10, 2473-2505.

[21] Shen, X. And HuAng, H. (2006). Optimal model assessment, selection, and combination, Journal of the American Statistical Association, 101, 554-568.

[22] Shen, X. AND Wong, W. (1994). Convergence rate of sieve estimates, Annals of Statistics, 22, 580-615.

[23] TAKeuchi, I., LE, Q. AND SEARS, T. (2009). Nonparametric conditional density estimation using piecewise-linear solution path of kernel quantile regression. Neural Computation, 21, 533-559.

[24] WahBA, G. (1990). Spline models for observational data, Philadelphia: SIAM.

[25] WAHBA, G. (2002). Soft and hard classification by reproducing kernel Hilbert space methods. In Proceedings of the National Academy of Sciences, 16524-16530.

[26] WANG, H., ZHU, Z., AND ZHOU, J. (2009). Quantile regression in partially linear varying coefficient models, Annals of Statistics, 37, 3841-3866.

[27] WAng, J. And Shen, X. (2006). Estimation of generalization error: random and fixed inputs, Statistica Sinica, 16, 569-588.

[28] WAng, J., Shen, X. And LiU, Y. (2008). Probability estimation for large margin classifiers, Biometrika, 95, 149-167. 
[29] Wu, T., Lin, C. AND WEng.R. (2004). Probability estimates for multi-class classification by pairwise coupling, Journal of Machine Learning Research, 5, 975-1005.

[30] WU, Y., ZHANG, H. AND LiU, Y. (2010). Robust model-free multiclass probability estimation, Journal of the American Statistical Association, 105, 424-436.

[31] Wu, Y. AND LIU, Y. (2009). Stepwise multiple quantile regression estimation using noncrossing constraints, Statistics and Its Interface, 2, 299-310.

[32] Xu, M., Watanachaturaporn, P., Varshney, P and Arora, M. (2005). Decision tree regression for soft classification of remote sensing data, Remote Sensing of Environment, 97, 322-336.

[33] YAng, Y. And HE, X. (2012). Bayesian empirical likelihood for quantile regression, The Annals of Statistics, 40, 1102-1131.

[34] Yu, K., LU, Z. ANd StAnder, J. (2003). Quantile regression: applications and current research areas, The Statistician, 52, 331-350.

[35] Zhou, D. (2002). The covering number in learning theory, Journal of Complexity, 18, 739767. 
Table 1: Simulated examples. Estimated means and standard deviations (in parentheses) of 1-norm, 2-norm, GKL loss and CEE for various estimation methods based on 50 replications.

\begin{tabular}{lcccc}
\hline \hline & 1-norm & 2-norm & EGKL & CEE \\
\hline Example 1 & & & & \\
\hline OUR & $0.316(0.0151)$ & $0.034(0.0034)$ & $0.080(0.0076)$ & $1.426(0.0122)$ \\
BLM & $0.447(0.0357)$ & $0.068(0.0082)$ & $0.211(0.0256)$ & $1.552(0.0329)$ \\
TREE & $0.404(0.0293)$ & $0.061(0.0091)$ & $0.178(0.0370)$ & $1.521(0.0403)$ \\
WMC & $0.336(0.0363)$ & $0.040(0.0089)$ & $0.139(0.0283)$ & $1.483(0.0354)$ \\
\hline Example 2 & & & & \\
\hline OUR & $0.359(0.0179)$ & $0.023(0.0026)$ & $0.104(0.0108)$ & $2.143(0.0168)$ \\
BLM & $0.468(0.0345)$ & $0.042(0.0048)$ & $0.245(0.0325)$ & $2.279(0.0406)$ \\
TREE & $0.511(0.0396)$ & $0.051(0.0095)$ & $0.256(0.0480)$ & $2.289(0.0514)$ \\
WMC & $0.560(0.0547)$ & $0.063(0.0151)$ & $0.270(0.0501)$ & $2.305(0.0597)$ \\
\hline Example 3 & & & & \\
\hline OUR & $0.412(0.0191)$ & $0.015(0.0016)$ & $0.136(0.0146)$ & $2.865(0.0166)$ \\
BLM & $0.505(0.0286)$ & $0.027(0.0030)$ & $0.307(0.0460)$ & $3.032(0.0542)$ \\
TREE & $0.714(0.0469)$ & $0.056(0.0084)$ & $0.352(0.0476)$ & $3.085(0.0527)$ \\
WMC & -- & -- & - & - \\
\hline Example 4 & & & & - \\
\hline OUR & $0.442(0.0163)$ & $0.072(0.0041)$ & $0.167(0.0100)$ & $1.496(0.0140)$ \\
BLM & $0.555(0.0194)$ & $0.114(0.0070)$ & $0.345(0.0324)$ & $1.676(0.0344)$ \\
TREE & $0.679(0.0539)$ & $0.169(0.0249)$ & $0.568(0.0855)$ & $1.901(0.0859)$ \\
WMC & $0.481(0.0510)$ & $0.084(0.0181)$ & $0.231(0.0468)$ & $1.561(0.0498)$ \\
\hline Example 5 & & & & \\
\hline OUR & $0.486(0.0188)$ & $0.044(0.0032)$ & $0.201(0.0144)$ & $2.217(0.0153)$ \\
BLM & $0.681(0.0209)$ & $0.098(0.0076)$ & $0.552(0.0558)$ & $2.573(0.0581)$ \\
TREE & $0.790(0.0556)$ & $0.124(0.0143)$ & $0.612(0.0807)$ & $2.621(0.0861)$ \\
WMC & $0.702(0.0584)$ & $0.095(0.0193)$ & $0.455(0.0889)$ & $2.472(0.0913)$ \\
\hline \hline
\end{tabular}


Table 2: Real applications. Estimated means and standard deviations (in parentheses) of CEE and MCE for various estimation methods base on 50 replications.

\begin{tabular}{lcr}
\hline \hline & CEE & MCE \\
\hline Iris example & & \\
\hline OUR & $0.167(0.0313)$ & $0.041(0.0224)$ \\
BLM & $2.164(0.0190)$ & $0.051(0.0260)$ \\
TREE & $0.220(0.0851)$ & $0.066(0.0234)$ \\
WMC & $0.146(0.0505)$ & $0.052(0.0245)$ \\
\hline Wine quality example & & \\
\hline OUR & $0.926(0.0125)$ & $0.468(0.0132)$ \\
BLM & $0.983(0.0227)$ & $0.510(0.0133)$ \\
TREE & $1.472(0.1215)$ & $0.530(0.0230)$ \\
WMC & $0.945(0.0226)$ & $0.490(0.0241)$ \\
\hline Abalone example $K=5$ & & \\
\hline OUR & $1.391(0.0121)$ & $0.640(0.0138)$ \\
BLM & $1.495(0.0483)$ & $0.661(0.0101)$ \\
TREE & $1.975(0.0694)$ & $0.732(0.0270)$ \\
WMC & $1.930(0.1440)$ & $0.675(0.0190)$ \\
\hline Abalone example $K=8$ & & \\
\hline OUR & $1.742(0.0123)$ & $0.721(0.0120)$ \\
BLM & $2.173(0.2202)$ & $0.775(0.0174)$ \\
TREE & $2.040(0.0834)$ & $0.749(0.0208)$ \\
WMC & -- & -- \\
\hline Abalone example $K=10$ & & \\
\hline OUR & $1.910(0.0203)$ & $0.756(0.0146)$ \\
BLM & $3.050(0.4905)$ & $0.834(0.0197)$ \\
TREE & $2.149(0.1130)$ & $0.771(0.0291)$ \\
WMC & -- & - \\
\hline \hline
\end{tabular}


Figure 1: A solution surface of $\hat{f}_{\lambda, \tau}$ as a function of $(\lambda, \tau)$ in a randomly selected replication of Example 1.

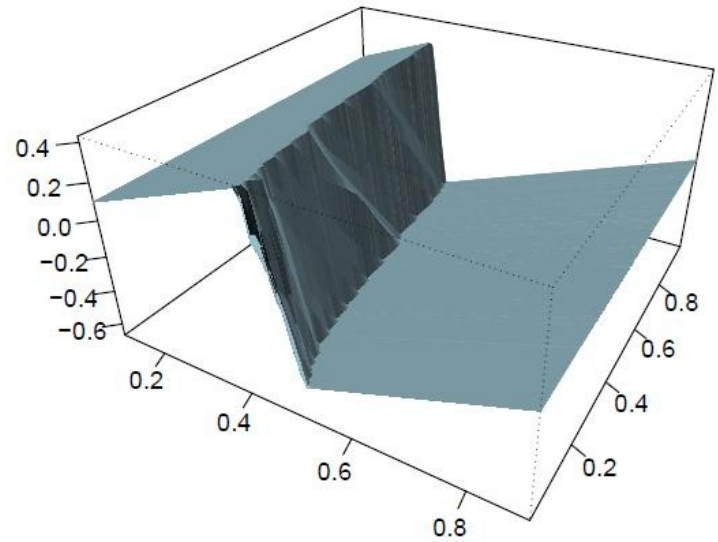

